\title{
STOCHASTIC INTEGRALS AND PARABOLIC EQUATIONS IN ABSTRACT WIENER SPACE
}

\author{
BY HUI-HSIUNG KUO ${ }^{1}$ AND M. ANN PIECH ${ }^{2}$ \\ Communicated by Lee A. Rubel, September 6, 1972
}

Kuo [2] has developed a theory of stochastic integrals and Piech [3] has established the existence of fundamental solutions of a class of parabolic equations, both working within the context of abstract Wiener space. In this note we establish the relationship between the work of Kuo and Piech, and as a consequence of this relationship we obtain a uniqueness theorem for fundamental solutions. We also provide a new proof of the nonnegativity and semigroup properties of fundamental solutions.

Let $H$ be a real separable Hilbert space, with inner product (, ) and norm $|\cdot|$; let $\|\cdot\|$ be a fixed measurable norm on $H$; let $B$ be the completion of $H$ with respect to $\|\cdot\|$; and let $i$ denote the natural injection of $H$ into $B$. The triple $(H, B, i)$ is an abstract Wiener space in the sense of Gross [1]. We may regard $B^{*} \subset H^{*} \approx H \subset B$ in the natural fashion. A bounded linear operator from $B$ to $B^{*}$ may thus be viewed as an operator on $B$ or, by restriction to $H$, as an operator on $H$. The restriction to $H$ of a member $T$ of $L\left(B, B^{*}\right)$ is of trace class in $L(H)(\equiv L(H, H))$ and

$$
\left\|T_{\mid H}\right\|_{\operatorname{Tr}} \leqq \text { constant } \cdot\|T\|_{L\left(B, B^{*}\right)} .
$$

Where no confusion of interpretation is possible, we will use $T$ for $T_{\mid H}$. In order to work with stochastic integrals on $(H, B, i)$ we formulate the following hypothesis:

(h) There exists an increasing sequence $\left\{P_{n}\right\}$ of finite dimensional projections on $B$ such that $P_{n}[B] \subset B^{*},\left\{P_{n}\right\}$ converges strongly to the identity on $B$, and $\left\{P_{n \mid H}\right\}$ converges strongly to the identity on $H$.

For $t>0$, let $p_{t}$ denote the Wiener measure on the Borel field of $B$ which is determined by Gauss cylinder set measure on $H$ of variance parameter $t$. Let $\Omega$ be the space of continuous functions $\omega$ from $[0, \infty)$ into $B$ and vanishing at zero, and let $\mathscr{M}$ be the $\sigma$-field of $\Omega$ generated by the functions $\omega \rightarrow \omega(t)$. Then there is a unique probability measure $\mathscr{P}$ on $\mathscr{M}$ for which the condition $0=t_{0}<t_{1}<\cdots<t_{n}$ implies that $\omega\left(t_{j+1}\right)-\omega\left(t_{j}\right)$, $0 \leqq j \leqq n-1$, are independent and $\omega\left(t_{j+1}\right)-\omega\left(t_{j}\right)$ has distribution

AMS(MOS) subject classifications (1970). Primary 28A40, 60H05, 35K 15.

Key words and phrases. Abstract Wiener space, stochastic integrals, parabolic equation, fundamental solution.

${ }^{1}$ Research supported by NSF grant GP-19617.

2 Research supported by NSF grants GP-1965i and GP-28934. 
measure $p_{t_{j+1}-t_{j}}$ in $B$. The process $W_{t}$ defined by $W_{t}(\omega) \equiv \omega(t)$ is called a Wiener process on $B$. The following theorem is a special case of Theorem 5.1 of $[2]$.

THEOREM 1. Assume that $C$ satisfies the following conditions:

(1-a) $C: B \rightarrow L(B)$;

(1-b) $C(x)-I$ has range in $B^{*}$ for all $x$ in $B$;

(1-c) $\|C(x)-C(y)\|_{H-S} \leqq$ constant $\cdot\|x-y\|_{B}$ for all $x$ and $y$ in $B$; where $\|\cdot\|_{H-S}$ is the Hilbert-Schmidt norm in $L(H)$;

$(1-\mathrm{d})\|C(x)-I\|_{H-S}^{2} \leqq$ constant $\cdot(1+\|x\|)^{2}$ for all $x$ in $B$. Then the stochastic integral equation

$$
X_{t}(\omega)=X_{0}(\omega)+\int_{0}^{t} C\left(X_{s}(\omega)\right) d W_{s}(\omega)
$$

possesses a unique continuous solution which is nonanticipating with respect to the family $\left\{\mathscr{M}_{t}\right\}$ where $\mathscr{M}_{t}$ is the $\sigma$-field generated by $\left\{W_{s}: 0 \leqq s \leqq t\right\}$. This solution is a homogeneous strong Markov process.

Assume that $f$ is a function with domain in $B$ and range in some Banach space $W$. The Fréchet derivative of $f$ at $x$ will be denoted by $f^{\prime}(x)$ and is a member of the space $L(B, W)$. The $H$-derivative of $f$ at $x$ will be denoted by $D f(x)$ and is the value at zero of the Fréchet derivative of the function $g: H \rightarrow W$ defined by $g(h) \equiv f(x+h)$.

We consider a differential operator of the form

$$
L_{x, t} u(x, t) \equiv \operatorname{trace}\left[A(x) D^{2} u(x, t)\right]-\partial / \partial t u(x, t)
$$

where $A: B \rightarrow L(H), u: B \times(0, \infty) \rightarrow R$ and $D$ denotes $H$-differentiability, for $t$ fixed. We say that $L_{x, t} u$ exists if the relevant derivatives exist and if $A(x) D^{2} u(x, t)$ is of trace class in $L(H)$. We may now state the results of [3].

THEOREM 2. Assume that $A(x)$ is of the form $I-B(x)$, where

$(2-a) B(x)$ is a symmetric member of $L(H)$ and there exists an $\varepsilon>0$ such that $B(x) \leqq(1-\varepsilon) I$ for all $x$ in $B$;

(2-b) there exists a symmetric Hilbert-Schmidt operator $E$ on $H$ such that $B(x)$ is of the form $E B_{0}(x) E$, where $B_{0}(x) \in L(H)$ and $\left\|B_{0}(x)\right\|_{L(H)} \leqq 1$ for all $x$ in $B$;

(2-c) $B_{0}^{\prime \prime}(x)$ exists and is a bounded uniformly Lip-1 function from $B$ to $L(B \rightarrow L(B \rightarrow L(H)))$;

(2-d) $\left|B_{0}^{\prime}(x)\right|_{L(B \rightarrow L(H))}$ is uniformly bounded;

(2-e) for any orthonormal basis $\left\{e_{i}\right\}$ of $H, \sum_{i}\left|B_{0}^{\prime}(x) e_{i}\right|_{L(H)}^{2}<$ constant, independently of $x$ in $B$.

Then there exists a family of finite real-valued signed Borel measures $\left\{q_{t}(x, d y): 0<t<\infty, \dot{x} \in B\right\}$ on $B$ such that if 


$$
q_{t} f(x) \equiv \int_{B} f(y) q_{t}(x, d y)
$$

then for each bounded real-valued uniformly Lip-1 function $f$ on $B$ we have $L_{x, t} q_{t} f(x)=0$ for all $x$ in $B$ and $t>0$. Moreover $\left\|q_{t} f-f\right\|_{\infty} \rightarrow 0$ as $t \downarrow 0$.

Assume henceforth that hypothesis (h) holds and that $A(x)$ satisfies (2-a)-(2-e). We require in addition that $B(x)$ is the restriction to $H$ of an operator which we also denote by $B(x)$ and which satisfies

(2-f) $B(\cdot): B \rightarrow L\left(B, B^{*}\right)$.

We may now regard $A(\cdot): B \rightarrow L(B)$. Then for each $x$ in $B A(x)_{\mid H}$ is positive definite and symmetric by $(2-\mathrm{a})$. Therefore $\left[A(x)_{\mid H}\right]^{1 / 2}$ exists as a member of $L(H)$. Moreover $I+\left[A(x)_{\mid H}\right]^{1 / 2}$ is invertible in $L(H)$. We define

$$
A(x)^{1 / 2} \equiv I-\left\{I+\left[A(x)_{\mid H}\right]^{1 / 2}\right\}^{-1} B(x) .
$$

It is easy to see that $A(x)^{1 / 2}$ satisfies (1-a), (1-c) and (1-d). (1-b) will follow once we establish that $\left[I+\left[A(x)_{\mid H}\right]^{1 / 2}\right]\left(B^{*}\right)=B^{*}$. Writing $\left[A(x)_{\mid H}\right]^{1 / 2}=$ $I-B(x)\left\{I+\left[A(x)_{\mid H}\right]^{1 / 2}\right\}^{-1}$ we see that $\left[A(x)_{\mid H}\right]^{1 / 2}$ maps $B^{*}$ to $B^{*}$ and $H \backslash B^{*}$ to $H \backslash B^{*}$. Since $I+\left[A(x)_{\mid H}\right]^{1 / 2}$ is invertible in $L(H)$ it follows that $\left[I+\left[A(x)_{\mid H}\right]^{1 / 2}\right]\left(B^{*}\right)=B^{*}$. Since $C(x) \equiv A(x)^{1 / 2}$ satisfies $(1-\mathrm{a})-(1-\mathrm{d})$ the stochastic integral equation

$$
X_{t}(\omega)=X_{0}(\omega)+\int_{0}^{t}\left[A\left(X_{s}(\omega)\right)\right]^{1 / 2} d W_{s}(\omega)
$$

has a unique solution $X_{t}$. We define

$$
r_{t}(x, d y) \equiv \mathscr{P}\left\{X_{t} \in d y: X_{0}=x\right\} .
$$

THEOREM 3. The fundamental solution $\left\{q_{t}(x, d y)\right\}$ of Theorem 1 coincides with the family $\left\{\boldsymbol{r}_{2 t}(x, d y)\right\}$ of transition probabilities associated with the solution of (1) and defined by (2). That is, $q_{t}(x, d y)=r_{2 t}(x, d y)$ for all $t>0$ and $x$ in $B$.

Proof. Two families of finite Borel measures on $B$ are identical if they act identically on all bounded real-valued uniformly Lip-1 functions $f$. That is, for any $\operatorname{such} f$, we must show that

$$
\int_{B} f(y) q_{t / 2}(x, d y)=\int_{B} f(y) r_{t}(x, d y) .
$$

We will write the left side of (3) as $q_{t / 2} f(x)$. Fix $\tau>0$. Define $F:[0, \tau) \times$ $B \rightarrow R$ by $F(t, x)=q_{(\tau-t) / 2} f(x)$. Then by Theorem 1 the function

$$
g(t, x) \equiv \partial / \partial t F(t, x)+\frac{1}{2} \operatorname{trace} A(x) D^{2} F(t, x)
$$

is identically zero on $[0, \tau) \times B$. It will be proved in a forthcoming paper 
[5] that, for each bounded real-valued Lip-1 function $f$ on $B$, the maps $(t, x) \rightarrow D\left(q_{t} f\right)(x)$ from $(0, \infty) \times B$ to $H$ with $|\cdot|$ and $(t, x) \rightarrow D^{2}\left(q_{t} f\right)(x)$ from $(0, \infty) \times B$ to the space of trace class operators on $H$ with trace class norm are continuous. This enables us to apply Ito's formula [2, Theorem 4.1] to $F(t, x)$, obtaining

$$
\begin{aligned}
F\left(t, X_{t}(\omega)\right)= & F(0, x)+\int_{0}^{t} g\left(s, X_{s}(\omega)\right) d s \\
& +\int_{0}^{t}\left\langle\left[A\left(X_{s}(\omega)\right)\right]^{1 / 2} D F\left(s, X_{s}(\omega)\right), d W_{s}(\omega)\right\rangle \\
= & q_{\tau / 2} f(x)+\int_{0}^{t}\left\langle\left[A\left(X_{s}(\omega)\right)\right]^{1 / 2} D F\left(s, X_{s}(\omega)\right), d W_{s}(\omega)\right\rangle
\end{aligned}
$$

for $0 \leqq t<\tau .\left\langle,>\right.$ denotes the $B^{*}-B$ pairing. By [2, (4) of Theorem 3.2] the expectation $(\mathscr{E})$ of the second term on the right side of (4) is zero. Thus

$$
\mathscr{E}\left[F\left(t, X_{t}(\omega)\right)\right]=q_{\tau / 2} f(x) .
$$

Letting $t \uparrow \tau$, we obtain

$$
\int_{B} f(y) r_{\tau}(x, d y)=\mathscr{E}\left[f\left(X_{\tau}(\omega)\right)\right]=q_{\tau / 2} f(x) .
$$

This establishes (3) and proves the theorem.

REMARK. Since the measures $\left\{q_{t}(x, d y)\right\}$ form the transition probabilities of a Markov process, it is an immediate consequence that $q_{s} q_{t} f(x)=$ $q_{s+t} f(x)$ (the "semigroup property") and that $q_{t}(x, d y)$ is a probability measure. These properties cannot be easily deduced from the work in [3]. They have been established in [4] in the presence of additional hypotheses of a technical nature on $A(x)$ (and in the absence of (2-f) and hypothesis (h)).

We note that, for the proof of Theorem 3, we have used only the properties of $q_{t} f$ mentioned in the statement of Theorem 2 together with smoothness properties of $D q_{t} f$ and $D^{2} q_{t} f$. We have thus proved the following uniqueness result for the fundamental solution of $L_{x, t} u=0$.

THEOREM 4. Assume that $L_{x, t}$ satisfies (2-a)-(2-f) and that $B$ satisfies hypothesis (h). Then the family $\left\{q_{t}(x, d y): t>0, x \in B\right\}$ whose existence is asserted by Theorem 2 is unique among families $\left\{\mu_{t}(x, d y): t>0, x \in B\right\}$ of bounded real-valued signed Borel measures on $B$ which satisfy the following requirements:

For each bounded real-valued uniformly Lip-1 function $f$ on $B$, setting $\mu_{t} f(x) \equiv \int_{B} f(y) \mu_{t}(x, d y)$,

(4-a) $\mu_{t} f(x)$ satisfies $L_{x, t} \mu_{t} f(x)=0$, 
(4-b) $\left\|\mu_{t} f-f\right\|_{\infty} \rightarrow 0$ as $t \downarrow 0$,

(4-c) $(t, x) \rightarrow D\left(\mu_{t} f\right)(x)$ and $(t, x) \rightarrow D^{2}\left(\mu_{t} f\right)(x)$ are continuous from $B \times(0, \infty)$ to $H$ and to the space of trace class operators on $H$ respectively.

\section{REFERENCES}

1. L. Gross, Abstract Wiener spaces, Proc. Fifth Berkeley Sympos. Math. Statist. and Probability (Berkeley, Calif., 1965/66), vol. II : Contributions to Probability Theory, part 1, Univ. of California Press, Berkeley, Calif., 1967, pp. 31-42. MR 35 \#3027.

2. H. H. Kuo, Stochastic integrals in abstract Wiener space, Pacific J. Math. 41 (1972), $469-483$

3. M. A. Piech, A fundamental solution of the parabolic equation on Hilbert space, J. Functional Analysis 3 (1969), 85-114. MR 40 \# 4815.

4. - A fundamental solution of the parabolic equation on Hilbert space. II: The semigroup property, Trans. Amer. Math. Soc. 150 (1970), 257-286. MR 43 \# 3847.

5. - Diffusion semigroups on abstract Wiener space, Trans. Amer. Math. Soc. 166 (1972), 411-430.

Courant Institute of Mathematical Sciences, New York University, New York, NEW YORK 10012

Department of Mathematics, State University of New York at Buffalo, AmHerst, NeW YORK 14226

Current address (Hui-Hsiung Kuo): Department of Mathematics, University of Virginia, Charlottesville, Virginia 22903 